\title{
Sommes nous en train de " boucler la boucle " pour répondre aux besoins thérapeutiques des patients des soins intensifs?
}

\author{
par Marc M. Perreault
}

DOI: 10.4212/cjhp.v74i3.3146

Les professionnels de la santé prodiguent des soins remarquables aux patients gravement malades, dès leur admission au service des urgences, jusqu'après leur transfert à l'unité des soins intensifs (USI) et, plus tard, au moment de leur retour dans la communauté. Heureusement pour ces patients, les taux de survie après un passage à l'USI ont augmenté. Nombre de patients souffrent toutefois de complications à court et à long terme à la suite de leur séjour dans une USI. Le syndrome post-soins intensifs (SPSI) se caractérise par une myriade de conséquences physiques, cognitives et psychosociales faisant suite aux maladies graves, qui empêchent les patients de retrouver leur capacité de fonctionnement antérieure, ce qui réduit leur qualité de vie et entraîne des détresses importantes chez les proches aidants ${ }^{1}$.

En 2016, le James Lind Alliance Priority Setting Partnership (Royaume-Uni) a reconnu ce syndrome comme l'une des deux priorités de recherche, non seulement pour les cliniciens des USI, mais aussi pour les patients des USI et les familles ${ }^{2}$. Des organismes, comme la Society of Critical Care Medicine, sous l'égide de la Thrive Collaboratives, mettent au point des initiatives visant à aborder le problème de la vie après la sortie de l'USI et à déterminer le modèle de soins le plus efficace pour les patients ayant quitté les soins intensifs ${ }^{3}$.

Des cliniques SPSI et des groupes de soutien par des pairs ont été mis en place pour répondre aux besoins des patients qui survivent à une maladie grave, et les pharmaciens commencent à assumer ce nouveau rôle au sein de cliniques multidisciplinaires. Selon moi, les pharmaciens spécialistes œuvrant en soins critiques sont bien placés pour contribuer aux soins de ces patients désormais ambulatoires dans ces cliniques. Ils connaissent non seulement le patient qui a séjourné à l'USI et les membres de sa famille, mais aussi les risques liés aux pharmacothérapies et aux complications, auxquels chaque patient pourrait être exposé.

Que pourrait-on attendre du pharmacien ouvrant dans une telle clinique? D'abord et avant tout, il pourrait effectuer un bilan comparatif exhaustif des médicaments ${ }^{4}$.
Ces patients reçoivent une panoplie de médicaments lors de leur admission à l'USI, mais après leur congé, bon nombre de ces médicaments ne sont plus indiqués. Malheureusement, ceux-ci sont encore souvent administrés pendant les transitions de soins et continuent parfois à l'être au moment du congé de l'hôpital. On notera par exemple l'amorce d'un traitement diurétique pour gérer la surcharge liquidienne, l'utilisation d'antagonistes $\beta$-adrénergiques pour prévenir la fibrillation auriculaire postopératoire ou l'administration d'antipsychotiques pour faire face aux périodes d'agitation à l'USI. En tant que membre de l'équipe de la clinique de SPSI, le pharmacien pourrait revoir la médication en cours et cesser les médicaments qui ne sont plus indispensables. Il pourrait aussi réévaluer le traitement qui était auparavant suivi à domicile et qui aurait pu être abandonné pendant l'hospitalisation et réinstaurer les médicaments requis afin d'éviter d'autres effets indésirables responsables de visites ou de réadmissions aux urgences.

Les interactions avec le patient et la famille dans cette clinique ambulatoire permettraient de sensibiliser davantage tous les professionnels de la santé au lourd tribut auquel les patients sont soumis après un séjour prolongé dans une USI. Le déconditionnement, la faiblesse musculaire, la déficience respiratoire, les douleurs chroniques, l'anxiété, les troubles du sommeil, les cauchemars et les troubles de stress post-traumatique sont fréquents et peuvent présenter des difficultés au quotidien pour les patients et les membres de leur famille. La gestion de ces conséquences négatives au sens large nécessite une approche multidisciplinaire et justifie le besoin de groupes de soutien par les pairs au sein desquels les patients et les membres des familles peuvent briser leur isolement et partager des préoccupations communes.

La pandémie de COVID-19 a compliqué l'organisation et le maintien de telles initiatives; cependant, du point de vue du patient, l'isolement résultant du confinement est une raison convaincante de les poursuivre. Je soupçonne que le nombre croissant de patients atteints du « Covid long », qui 
souffrent de divers symptômes débilitants des mois après leur infection initiale et leur séjour à l'USI, se rendront régulièrement dans de telles cliniques.

La mise en place de cliniques SPSI dotées d'un personnel hautement motivé s'effectue actuellement à très petite échelle au Canada. La connaissance des résultats cliniques des patients associés à de telles initiatives est actuellement limitée, mais cette lacune est en voie de résolution ${ }^{5,6}$. La collaboration d'un pharmacien spécialiste œuvrant en soins critiques au sein de l'équipe de la clinique SPSI est cruciale.

Il faut parvenir à une meilleure définition d'un modèle de soins plus efficace pour traiter les patients qui ont quitté les soins intensifs. Les cliniques SPSI nous permettraient de «boucler la boucle " pour répondre aux besoins thérapeutiques des patients gravement malades. Cependant, en attendant que le rôle de ces cliniques soit mieux cerné et qu'elles deviennent monnaie courante, assurons-nous qu'un pharmacien spécialiste œuvrant en soins critiques examine toutes les ordonnances de médicaments au moment du congé de l'USI, qu'il rédige un plan visant à reprendre ceux qui sont nécessaires et à abandonner ceux qui sont superflus.

\section{Références}

1. Needham DM, Davidson J, Cohen H, Hopkins RO, Weinert C, Wunsch $\mathrm{H}$, et al. Improving long-term outcomes after discharge from intensive care unit: report from a stakeholders' conference. Crit Care Med. 2012;40(2):502-9.
2. Arulkumaran N, Reay H, Brett SJ; JLA Intensive Care Research Priority Setting Partnership. Research priorities by professional background - a detailed analysis of the James Lind Alliance Priority Setting Partnership. J Intensive Care Soc. 2016;17(2):111-6.

3. Haines KJ, McPeake J, Hibbert E, Boehm LM, Aparanji K, Bakhru RN, et al. Enablers and barriers to implementing ICU follow-up clinics and peer support groups following critical illness: the Thrive Collaboratives. Crit Care Med. 2019;47(9):1194-200.

4. Stollings JL, Bloom SL, Wang L, Ely EW, Jackson JC, Sevin CM. Critical care pharmacists and medication management in an ICU recovery center. Ann Pharmacother. 2018;52(8):713-23.

5. Bloom SL, Stollings JL, Kirkpatrick O, Wang L, Byrne DW, Sevin CM, et al. Randomized clinical trial of an ICU recovery pilot program for survivors of critical illness. Crit Care Med. 2019;47(10):1337-45.

6. Akhlaghi N, Needham DM, Bose S, Banner-Goodspeed VM, Beesley SJ, Dinglas VD, et al. Evaluating the association between unmet healthcare needs and subsequent clinical outcomes: protocol for the Addressing Post-Intensive Care Syndrome-01 (APICS-01) multicentre cohort study. BMJ Open. 2020;10(10):e040830.

Marc M. Perreault, B. Pharm., M. Sc., Pharm. D., FCSHP, FOPQ, est pharmacien en soins intensifs à l'Hôpital général de Montréal [Québec]. Il est aussi rédacteur adjoint pour le Journal canadien de la pharmacie hospitalière.

Conflits d'intérêts : Aucune déclaration.

Adresse de correspondance :

$D^{r}$ Marc Perreault

Service de pharmacie

Hôpital général de Montréal

1650, av. Cedar, bureau C1-200

Montréal QC H3G 1A4

Courriel : marc.perreault@umontreal.ca

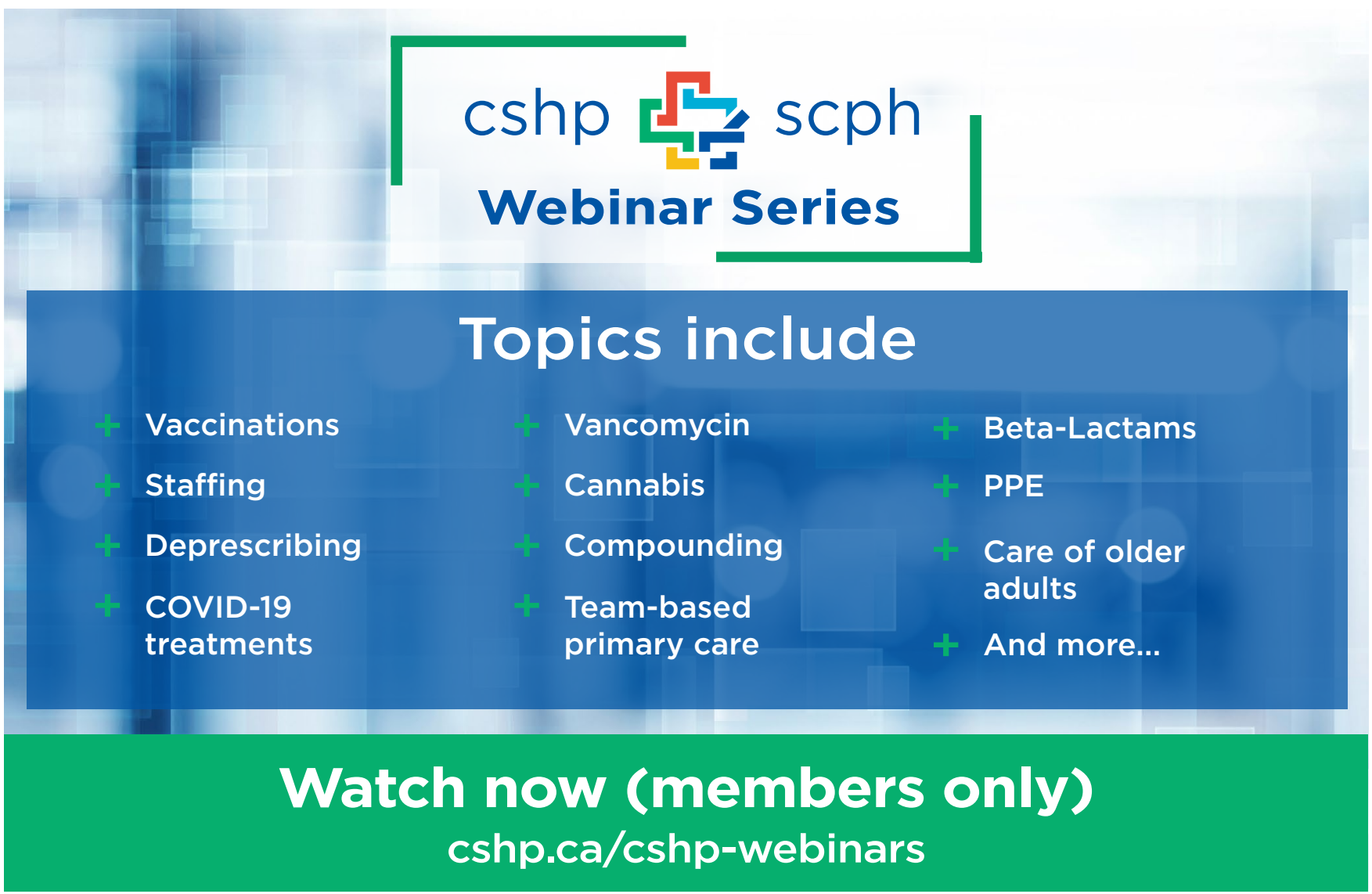

